\title{
1 A Study of the Pressure Profiles near the First Pumping Aperture in a High
}

2 Pressure Photoelectron Spectrometer

3 J. Matthias Kahk, ${ }^{1}$ Ignacio J. Villar-Garcia, ${ }^{1}$ Lorenza Grechy, ${ }^{2}$ Paul J. K. Bruce, ${ }^{2}$ Peter E. Vincent, ${ }^{2}$ Susanna K. Eriksson, ${ }^{3}$ Håkan Rensmo, ${ }^{4}$ Maria Hahlin, ${ }^{4}$ John Åhlund, ${ }^{5}$ Mårten O. M. Edwards, ${ }^{5}$ and David J. Payne ${ }^{1 *}$

1. Department of Materials, Imperial College London, Exhibition Road, London, SW7 2AZ, UK

2. Department of Aeronautics, Imperial College London, SW7 2AZ, United Kingdom

${ }^{3 .}$ Department of Chemistry-Ångström, Uppsala University, Box 523, 75120 Uppsala, Sweden

4. Department of Physics and Astronomy, Uppsala University, Box 516, 75120 Uppsala, Sweden

${ }^{5 .}$ VG Scienta AB, Box 15120, 75015 Uppsala, Sweden

*Corresponding author: d.payne@imperial.ac.uk

Abstract

In a high-pressure photoelectron spectrometer, the sample is positioned close to a differential pumping aperture, behind which the pressure is several orders of magnitude lower than the pressure in the analysis chamber. To find the optimal sample position, where the path length of the photoelectrons through the high pressure region is minimized as far as possible without compromising knowledge of the actual pressure at the sample surface, an understanding of the pressure variations near the sample and the aperture is required. A computational fluid dynamics study has been carried out to examine the pressure profiles, and the results are compared against experimental spectra whose intensities are analyzed using the Beer-Lambert law. The resultant pressure profiles are broadly similar to the one previously derived from a simplistic molecular flow model, but indicate that as the pressure in the analysis chamber is raised, the region over which the pressure 


\section{Introduction}

Photoelectron Spectroscopy (PES) is a versatile technique for determining the elemental composition and electronic structure of materials [1]. In typical usage, PES is performed under conditions of high or ultrahigh vacuum. This is required in order to minimize the attenuation of the emitted photoelectrons on their path from the sample to the detector. It is also essential for the operation of electron energy analyzers, in which electro-optical elements with voltages up to several $\mathrm{kV}$ are applied to selectively detect photoelectrons of a particular kinetic energy. Whilst this requirement makes PES ideally suited for studies of the fundamental surface science of ultraclean surfaces, it poses severe limitations on the use of the technique to study systems of industrial, biological or environmental relevance, where the surface chemistry typically occurs at much higher pressures. To overcome these limitations, strategies have been developed which allow the operation of photoelectron spectrometers at pressures of up to tens of millibars $[2,3,4]$.

Historically, the first steps towards modern high pressure photoelectron spectroscopy (HiPPES) were taken in the groups of Kai and Hans Siegbahn, where instruments for measuring photoelectron spectra of gases and liquids were developed $[5,6]$. The method was extended to the study of solids under a controlled gas atmosphere of up to 1 mbar by W. Roberts [7], and the modern generation of instruments with an electrostatic lens incorporated into the differential pumping system was introduced by Bluhm et al. in 2002 [8].

A common feature of all of the designs is the use of one or several differential pumping stages to maintain the large pressure difference of up to 11 orders of magnitude between the sample chamber and the analyzer. The sample is placed near the entrance aperture of the first differential pumping stage, in order to minimize the attenuation of the signal due to inelastic scattering of the photoelectrons by the gas molecules. (Figure 1.) However, if the sample is brought too close to the aperture, the local pressure at the sample surface will differ from the value measured in the back of the chamber, and there may also exist a pressure gradient along the plane of the sample. Both of these effects complicate the analysis of the data considerably, and are thus

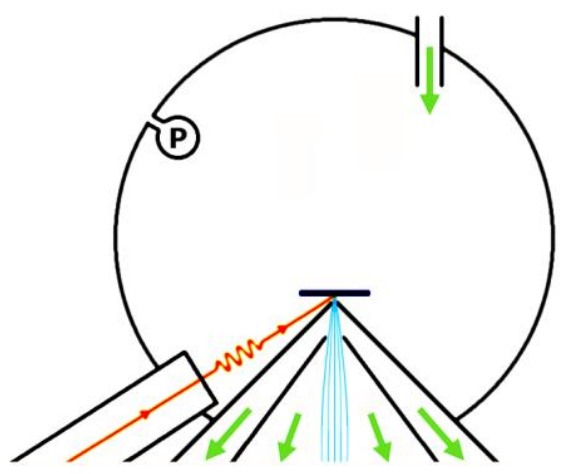

Figure 1: A schematic diagram of a high-pressure photoelectron spectrometer. The incoming X-rays (orange wiggly line) impinge the sample, resulting in the emission of photoelectrons (blue lines), which travel through several differential pumping stages and are eventually detected by an electron energy analyser. Gas is allowed to enter at the back of the chamber through a leak valve, and is pumped out through the entrance aperture of the differential pumping system (cone). The green arrows indicate the inlet (leak valve) and outlet (turbo pump) positions. The pressure in the analysis chamber is monitored at the back of the chamber far from the inlet and far from the sample position. 
best avoided. It is therefore necessary to determine the optimal position of the sample during measurements, where the attenuation of the photoelectron signal is minimized as far as reasonably possible, without compromising the required level of knowledge or control of the actual pressure at the sample surface.

Presently, the experimental practise follows the analysis presented in the paper by Ogletree et al [8], who derived the centre-line pressure profile for flow through a circular orifice in an infinitely thin plane, under the conditions of pure molecular flow [Equation 1].

$$
p(l)=\frac{1}{2} p_{0}\left(1-\frac{l}{\sqrt{1+l^{2}}}\right)
$$

Here, $p(l)$ denotes the pressure at distance $l$, where the positive direction of $l$ points towards the analyser. $p_{0}$ is the pressure at the back of the analysis chamber.

It is instructive to consider, whether the application of the molecular flow regime is appropriate for the experimental situation in modern spectrometers. The transition from free molecular flow to macroscopic continuum flow is described by the Knudsen number, $K n$, defined as the ratio between the mean free path of the gas molecules, $\lambda$, and the characteristic length scale of the flow, $L[9]$.

$$
K n=\frac{\lambda}{L}
$$

For $K n<0.01$, i.e. when the mean free path of the molecules is much smaller than the characteristic length scale, the continuum formulation of fluid dynamics holds and the Navier-Stokes equations can be used to model the flow [9], In contrast, when $K n>10$, collisions between gas molecules are rare, and the physics of free molecular flow apply. Intermediate Knudsen numbers are more difficult to treat, although in the range $0.01<K n<0.1$ the Navier-Stokes equations can still yield reasonable results, if the no-slip boundary condition at solid surfaces is relaxed. This is the so-called "slip flow" regime [9].

In a high pressure photoelectron spectrometer, the characteristic dimension can be taken as the diameter of the aperture, which is here chosen to be $0.3 \mathrm{~mm}$. The operating pressures of interest are in the millibar range. The mean free path of nitrogen molecules can be obtained from Equation 3, and is equal to $68 \mu \mathrm{m}$ at $1 \mathrm{mbar}$ and $2.3 \mu \mathrm{m}$ at $30 \mathrm{mbar}[10]$.

$$
l=\frac{k_{B} T}{\sqrt{2} \pi d^{2} p}
$$

$l$ denotes the mean free path, $k_{B}$ is the Boltzmann constant, $T$ is the absolute temperature, $\pi d^{2}$ is the collision cross-section of the molecule and $p$ is the pressure.

The obtained Knudsen numbers are 0.23 at 1 mbar and 0.01 at 30 millibars, indicating that the assumption of free molecular flow is probably not very accurate, and that at the highest operating pressures the continuum flow models should be expected to yield reasonable results. Numerical simulations of flows and pressure profiles based on the continuum equations can be readily performed using commercial computational fluid dynamics packages, and via the use of partial slip boundary conditions it is also possible to similarly model slip-flow. Such simulations also allow one to consider more realistic geometries for the flows than a circular 
orifice in an infinitely thin plane. Indeed, in a real photoelectron spectrometer the aperture is located at the end of a cone, rather than in a flat wall; the walls of the cone also have a finite thickness, and the flow through the aperture can be affected by the presence of the sample and the sample holder in the near vicinity.

There have also been some previous efforts to experimentally verify the true pressure at the sample surface during a high-pressure photoemission experiment. The first of the reported methods is based on observing the adsorption-desorption isobars of the condensation of a gas on the sample. If the pressure/temperature behaviour of the process being studied is known, the pressure at the sample surface can be estimated from the apparent behaviour, and compared to the background pressure measured in the chamber. In the paper titled "Controlled-Atmosphere Photoelectron Spectroscopy", Grunze et al. recorded the temperatures at which ice multilayers are formed at different background pressures and compared them to the vapour pressure of water at those temperatures [11]. The real pressure at the sample was estimated to be approximately an order of magnitude lower than the pressure at the manometer. This relatively large difference could be explained by the fact that the sample-to-aperture distance used $(1.2 \mathrm{~mm})$ was relatively small compared to the size of the aperture $(5.4 \mathrm{~mm} \times 0.89 \mathrm{~mm})$ in the instrument used. The authors also pointed out the effect of cooling of the gas in the vicinity of the sample surface by heat transfer to the liquid nitrogen-cooled sample rod.

The second type of method, also used in reference [11], is based on an adaptation of the Beer Lambert law for the attenuation of electrons passing through a gaseous atmosphere:

$$
I=I_{0} e^{-\frac{p d}{\lambda}}
$$

where $I_{0}$ is the original intensity, $I$ is the transmitted intensity, $p$ is the pressure, $d$ is the path length of the electrons through the gas, and $\lambda$ is the electron mean free path. They recorded core level spectra at different background pressures, and then calculated the mean free paths from the slope of a plot of $-\ln \left(I / I_{0}\right)$ against pressure. Under the assumption that the attenuation of the elastic photoelectron flux is solely due to ionization processes, ionization cross-sections were also obtained for different gases, which could in turn be compared against literature values. However the formulation of the Beer Lambert law given in Equation 4 is directly applicable for regions of constant pressure only. For a region of varying pressure, the same formulation can only be used if the concept of an effective path length is introduced, but in general, the effective path length that the electrons traverse through the gaseous atmosphere is not known a priori. Effective path lengths can themselves be obtained from experimental data using Equation 4, if the nominal pressure and a tabulated mean free path for photoelectrons of a given kinetic energy are used, but this procedure does not yield any information about the actual pressure at the sample surface $[12,13]$.

\section{Theoretical methods}

We have performed theoretical simulations of the flow through the entrance aperture of the differential pumping setup of a high pressure photoelectron spectrometer using the commercial computational fluid dynamics package Star-CCM+ v.9.04.011 (CD-Adapco). The geometry used for the simulations was based on an axisymmetric model, whose cross-section is shown in Figure 2. In the geometry used, a wall boundary 
is placed in the close vicinity of the aperture, and flow is only allowed to enter from the sides. The wall boundary represents the sample, which is assumed to be much larger than the size of the aperture, as is the case in most high-pressure photoemission experiments.

The size of the simulation cell was always chosen to be considerably larger than the region with steep pressure gradients, to ensure that it would not affect the outcome of the simulation in the area of interest (i.e. near the cone). It was verified that enlarging the simulation cell further did not affect the outcome of the simulation.

The symmetry about the axis was also used as a boundary condition for the simulations, i.e. all of the simulations were performed in axisymmetric space. The implicit unsteady time integration scheme with a time step of $10^{-4} \mathrm{~s}$ was used, and the centre line pressure profiles converged to a steady state in all simulations. The results given in this paper are for calculations where the laminar flow model was used, but the Reynolds stress turbulence model with quadratic pressure strain was also tested, and the calculated pressures along the symmetry axis differed by less than $1 \%$ of the base pressure. The use of the laminar flow model was further justified a posteriori by the results of the simulation at $30 \mathrm{mbar}$, from which a Reynolds number of 290 can be calculated for flow through a $0.3 \mathrm{~mm}$ aperture. The coupled flow solver was used, and the fluid was modelled as an ideal gas with the physical parameters of nitrogen.

The gas inlet type used was a stagnation inlet, and the outlet type used was a pressure outlet. The pressure at the inlet was set to $30 \mathrm{mbar}, 9.4 \mathrm{mbar}$ or $5.0 \mathrm{mbar}$ as required. The pressure at the outlet was set to $10^{-3}$ mbar, which is similar to the actual pressures measured in the 1st differential pumping stage during high pressure experiments $[2,3,4]$. Varying the outlet pressure in the range $10^{-4} \mathrm{mbar}-3 \times 10^{-3} \mathrm{mbar}$ had a negligible effect on the obtained pressure profiles near the sample region. The temperature at the inlet was set to $300 \mathrm{~K}$ in all simulations. To ensure numerical stability a minimum allowable pressure for one simulation cell was set to $10^{-4}$ mbar. Lower values down to $10^{-8}$ mbar were tested and did not affect the outcomes of the simulations, when stable, but frequently lead to the divergence of the solution.

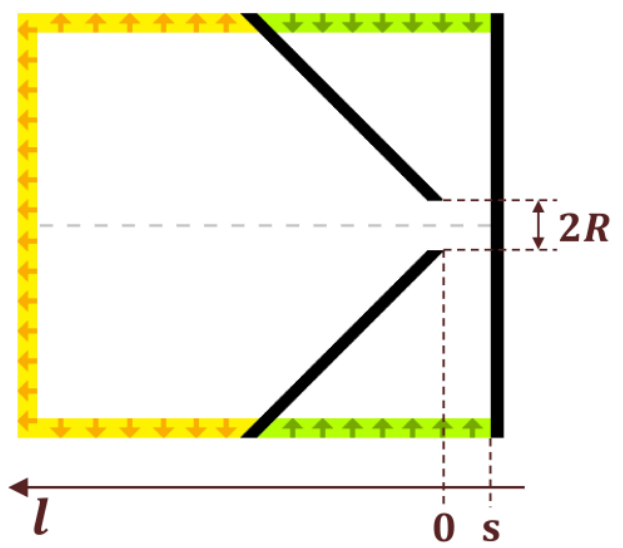

Figure 2: A 2D projection of the geometry used in the simulations. The green walls with inwards arrows are inlet boundaries, the yellow walls with outwards arrows are outlet boundaries, and the black walls, corresponding to the cone and the sample, are wall boundaries. The dashed horizontal line through the centre denotes the symmetry axis. 
The trimmer and prism layer meshers were used for generation of the volume mesh. The base size of the mesh was $0.05 \mathrm{~mm}$, and two additional levels of volumetric controls were used to create a finer mesh in the close vicinity of the aperture, such that at the aperture plane, the cell size was $0.0125 \mathrm{~mm}$. A finer mesh with a base size of $0.03 \mathrm{~mm}$ was also tested to ensure the resolution of the mesh did not affect the outcome of the simulation. The thickness of the prism layer was set to $100 \%$ of the base size, and 6 prism layers with a scaling factor of 1.1 were used. The combination of the volumetric controls and the prism layer mesher ensured that there were approximately 36 cells across the width of the aperture.

Two types of wall boundary conditions were employed. For simulations using the macroscopic continuum flow assumption, a no-slip boundary condition is applied, forcing the layer of fluid in immediate contact with the wall to have zero tangential velocity. For slip flow simulations, this no-slip boundary condition was relaxed. The implementation of slip-flow simulations in Star-CCM+ is described next.

Relaxing the no-slip boundary condition at solid surfaces results in a discontinuity in the velocity at the surface, which is described by Maxwell's slip equation (Equation 5) [9].

$$
U_{w a l l}=\left.\frac{2-\sigma}{\sigma} \lambda \frac{\partial U}{\partial y}\right|_{y=0}
$$

where $U_{\text {wall }}$ is the tangential slip velocity, $\sigma$ is the tangential momentum accommodation coefficient, $\lambda$ is the mean free path and $\mathrm{y}$ is the distance normal to the wall. Instead of $\sigma$, a "slip coefficient" $\alpha$ is sometimes used, such that $\alpha=(2-\sigma) / \sigma$ [14]. A somewhat different formulation of the slip equation is used in the present work:

$$
U_{\text {wall }}+\left.m K n \frac{\partial U}{\partial n}\right|_{n=0}=0
$$

Where $K n$ is the Knudsen number and $m$ is a positive constant characterizing the molecular interaction of the gas with the wall. However, these formulations become equivalent by setting $y=-n$ and $m=\alpha L$, where $L$ is the characteristic length scale of the flow. Following the recommendations of references [14] and [15], a value of $\alpha=1.15$ was used for the slip coefficient, corresponding to a tangential momentum accommodation coefficient of approximately $\sigma=0.93$.

For the implementation of slip-flow in Star-CCM+, it is also necessary to also consider the relationship between the tangential velocity at the wall surface, and the tangential velocity at the centre of a nearby simulation cell. It is assumed that for sufficiently small distances from the surface, the derivative of the tangential velocity component with respect to the distance normal to the surface is constant. In that case,

$$
\begin{gathered}
U_{\text {cell }}=U_{\text {wall }}+\left.n_{\text {cell }} \frac{\partial U}{\partial n}\right|_{n=0} \\
U_{\text {cell }}=-\left.m K n \frac{\partial U}{\partial n}\right|_{n=0}+\left.n_{\text {cell }} \frac{\partial U}{\partial n}\right|_{n=0}
\end{gathered}
$$

Noting that the positive direction of $\mathrm{n}$ points into the wall, $n_{\text {cell }}=-\Delta n$, where $\Delta n$ is the separation between the centroid of the cell and the wall. 


$$
\begin{gathered}
U_{\text {cell }}=\left.(-m K n-\Delta n) \frac{\partial U}{\partial n}\right|_{n=0}=(-m K n-\Delta n) \frac{U_{\text {wall }}}{-m K n} \\
U_{\text {wall }}=\frac{m K n}{\Delta n+m K n} U_{\text {cell }}=\frac{m K n / \Delta n}{1+(m K n / \Delta n)} U_{\text {cell }}
\end{gathered}
$$

For any particular simulation, values of the Knudsen number and the constant $c$ are first determined. Then, a vector field function is created, which relates the tangential velocity in a cell close to the wall to the tangential wall velocity. This vector field function is then applied as the tangential velocity specification to the wall boundaries along which slip-flow is simulated.

\section{Experimental methods}

The experimental apparatus used for the photoemission measurements has been previously described in detail in reference [3]. It consists of a Scienta MX $650 \mathrm{HP}$ monochromated Al Ka X-ray source $(\mathrm{h} v=$ $1486.6 \mathrm{eV}$ ), a stainless steel analysis chamber with a $\mu$-metal liner, a load lock chamber, and a Scienta R4000 HiPP-2 electron energy analyzer. The monochromator is mounted at an angle of $62.5^{\circ}$ relative to the symmetry axis of the analyzer; it is separated from the analysis chamber by a reinforced aluminium window which is located at a distance of $102 \mathrm{~mm}$ from the focal point on the sample. The size of the X-ray spot at the measurement position, as given by the horizontal and vertical $12 / 88$ widths, is $4.7 \times 1.4 \mathrm{~mm}$. The polycrystalline Ag and Au foils (99.95\% from A. Rasmussen a.s. and 99.99\% from KarAna AB); are mounted on a sample stage connected to a VG Scienta Transax four-axis manipulator. During high pressure measurements the analysis chamber was filled with nitrogen (99.999 \% Alphagaz $1^{\mathrm{TM}}$ from Air Liquide AB) using a needle valve to control the flow. To determine the distance between the sample and the entrance aperture, the sample is first moved right up to the aperture, whilst the X-ray source is on and one of the core level XPS peaks from the sample is monitored. When the analyzer count rate drops to zero, it is assumed that the sample is touching, or very close to touching the aperture. At the same time, the sample and the cone can be visually monitored through a view port in the analysis chamber. Then, using the manipulator the sample is retracted to the desired position. If this procedure is followed carefully, the real distance between the sample and the aperture should always be slightly larger than the measured distance, as the count rate will drop to noise level due to almost all of the X-ray beam being blocked just before the sample and the aperture come into physical contact.

\section{Results and discussion}

In Figures 3-6, the pressure profiles, 2D pressure maps, 2D Mach number maps and 2D velocity maps are shown for two simulations: one using the no-slip boundary condition, and the other using the Maxwell slip boundary condition. The other models and parameter values used in the simulations are listed in Table 1.

In Figure 3 the pressure profile from Ogletree et al. has also been displayed for comparison. As expected, at 30 mbar, the results of the simulations using the no-slip boundary condition or the Maxwell slip boundary condition are very similar. The difference in the boundary conditions can be best seen in the zoomed version of Figure 6, where a stationary fluid layer (blue in colourmap) against the aperture wall is present for the noslip simulations, but not in the other case. However, the calculated centre-line pressure profiles do clearly 


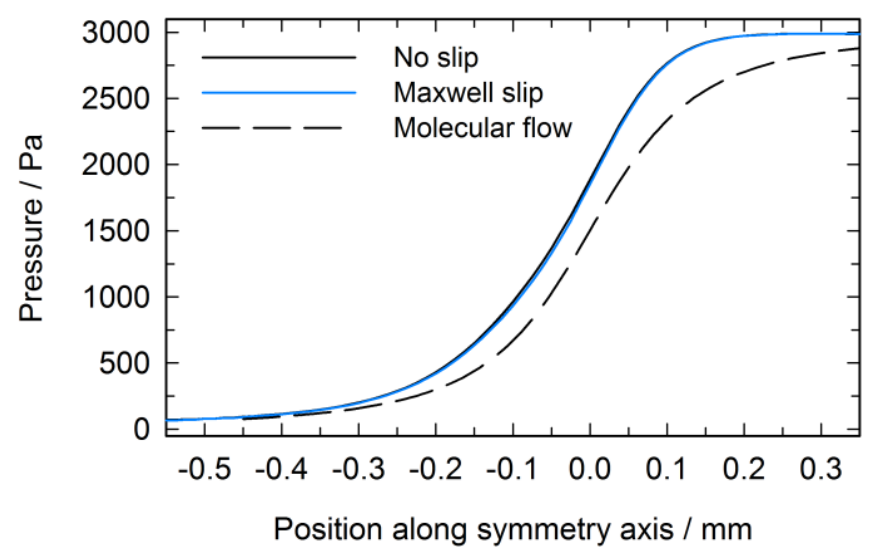

Figure 3: Pressure profiles along the axis of symmetry obtained using different tangential velocity boundary conditions for wall boundaries.
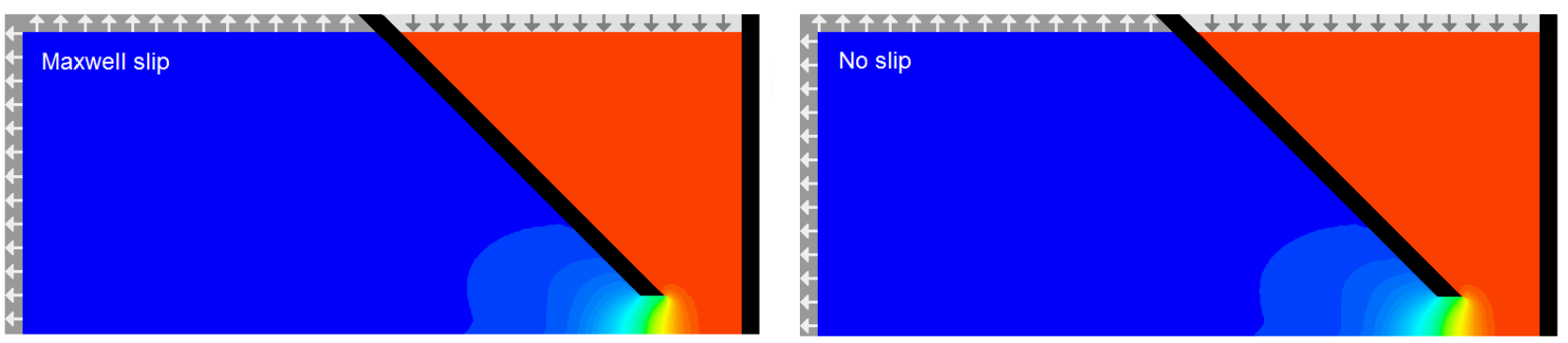

Absolute Pressure (mbar)

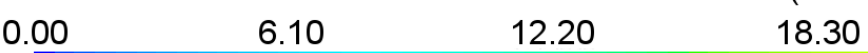

24.40

30.50

Figure 4: 2D pressure maps obtained using different tangential velocity boundary conditions for wall boundaries.

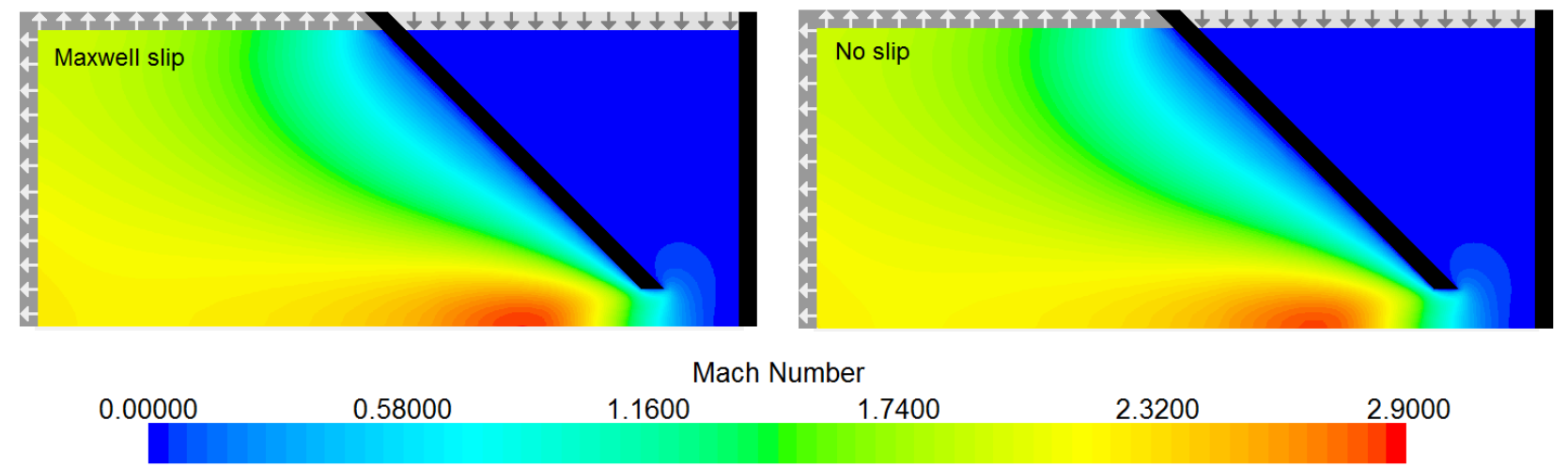

Figure 5: 2D Mach number maps obtained using different tangential velocity boundary conditions for wall boundaries.

differ from that obtained for molecular flow in the idealized geometry. For the slip-flow simulation at 30 mbar, the pressure at the aperture plane is $18.59 \mathrm{mbar}$, or $62.0 \%$ of the base pressure; and the pressure at the sample surface at the point of the symmetry axis is 29.85 mbar, or $99.5 \%$ of the base pressure. A comparison of the results of the slip-flow simulations at three different pressures and a range of sample-tocone distances is given in Table 2.

Attention is now turned to the experimental verification of the calculated pressure profiles. For this, a simple method is used that involves monitoring the intensity of a specific photoemission peak at high 

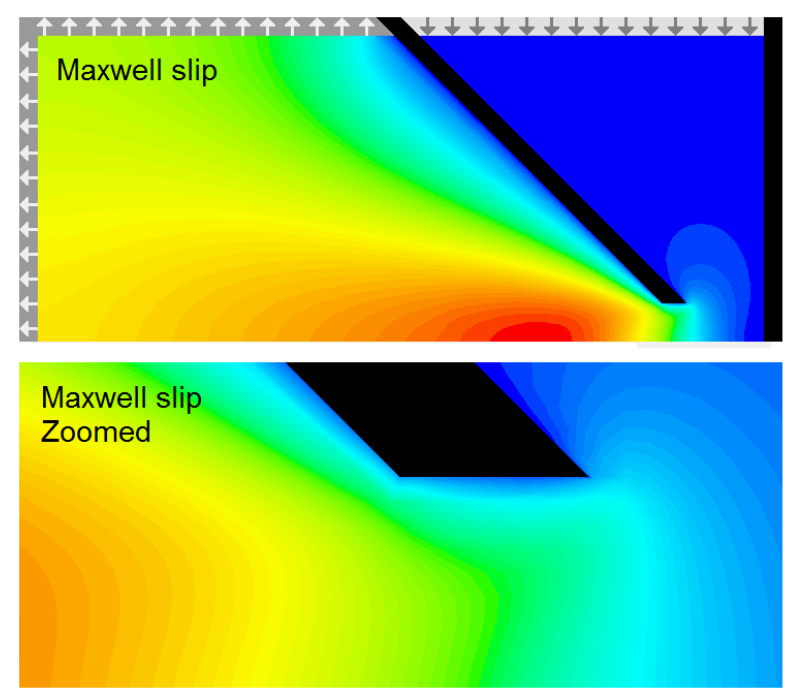

Velocity: Magnitude $(\mathrm{m} / \mathrm{s})$

0.00000 122.00

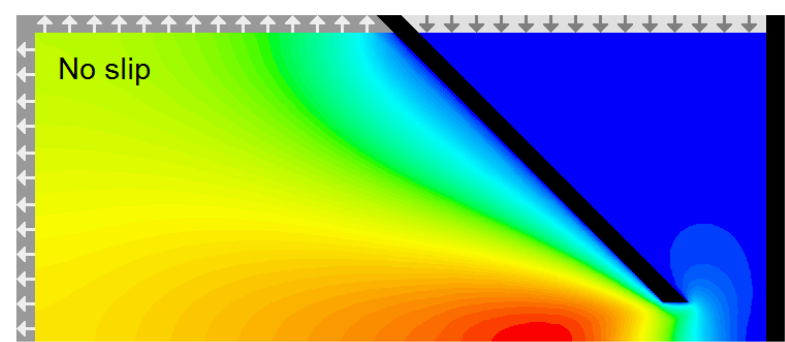

No slip

Zoomed

Figure 6: $2 \mathrm{D}$ velocity maps obtained using different tangential velocity boundary conditions for wall boundaries. In the zoomed in images one can observe the contrasting behaviour in the close vicinity of the cone aperture: for the no slip case, a stationary fluid layer is maintained against the aperture walls, whereas in the Maxwell slip case, no such stationary layer is present.

pressure, as the distance between the sample under study and the first aperture of the differential pumping system is varied. This dependence of the intensity on the position of the sample contains information about the attenuation of the photoelectron signal when travelling through the gas phase, which can be analyzed using an adapted version of the Beer-Lambert law. A series of spectra measured at different sample positions, showing the $\mathrm{Ag} 3 \mathrm{~d}_{5 / 2}$ core level peak of a polycrystalline silver foil measured under 5 mbar nitrogen atmosphere, are shown in Figure 7. It is evident that the intensity of the photoemission peak depends strongly on the position of the sample, but this dependence is in general rather complex as it results from a combination of multiple factors, including the intensity of the incident X-ray beam, the collection efficiency of the electron energy analyzer and, in the case of operation under high pressure, the attenuation of both the incident X-rays as well as the emitted photoelectrons as they travel through a high pressure atmosphere. To eliminate the influence of "instrumental" factors, a similar series of spectra was acquired under high vacuum conditions, where attenuation of the signal due to scattering can be assumed to be negligible, keeping other parameters (sample position, beam intensity and alignment, acquisition parameters) unchanged. All of the differences between the spectra at high vacuum and elevated pressures can then be

Table 1 - Physics models and parameter values used in the simulations shown in Figures 4-6

\begin{tabular}{ll}
\hline Sample-to-cone distance & $0.3 \mathrm{~mm}$ \\
Aperture width & $0.3 \mathrm{~mm}$ \\
Pressure outlet conditions & $10^{-3} \mathrm{mbar}, 300 \mathrm{~K}$ \\
Stagnation inlet conditions & $30 \mathrm{mbar}, 300 \mathrm{~K}$ \\
Minimum cell pressure & $10^{-4} \mathrm{mbar}$ \\
Flow physics model & Coupled flow \\
Gas physics model & Ideal gas \\
Turbulence specification & Laminar \\
\hline
\end{tabular}


Table 2 - Results of the slip flow simulations for different base pressures and sample-to-cone distances

\begin{tabular}{llllllll}
\hline Base pressure & \multicolumn{3}{c}{5.0 mbar } & & 9.4 mbar & \multicolumn{3}{c}{30 mbar } \\
\hline Sample position $(\mathrm{mm})$ & $\begin{array}{l}\text { Sample } \\
\text { position }(\mathrm{R})\end{array}$ & $\begin{array}{l}\text { p at sample } \\
\text { (mbar) }\end{array}$ & $\begin{array}{l}\text { \% of } \\
\text { base p }\end{array}$ & $\begin{array}{l}\text { p at sample } \\
\text { (mbar) }\end{array}$ & $\begin{array}{l}\text { \% of } \\
\text { base p }\end{array}$ & $\begin{array}{l}\text { p at sample } \\
\text { (mbar) }\end{array}$ & \% of base p \\
\hline $0.15 \mathrm{~mm}$ & 1.00 & 4.23 & 84.6 & 8.55 & 90.9 & 28.88 & 96.3 \\
$0.20 \mathrm{~mm}$ & 1.33 & 4.60 & 92.1 & 8.99 & 95.6 & 29.50 & 98.3 \\
$0.25 \mathrm{~mm}$ & 1.67 & 4.77 & 95.4 & 9.18 & 97.6 & 29.74 & 99.1 \\
$0.30 \mathrm{~mm}$ & 2.00 & 4.85 & 97.0 & 9.26 & 98.5 & 29.85 & 99.5 \\
$0.40 \mathrm{~mm}$ & 2.67 & 4.93 & 98.7 & 9.34 & 99.4 & 29.95 & 99.8 \\
$0.60 \mathrm{~mm}$ & 4.00 & 4.98 & 99.5 & 9.39 & 99.9 & 29.99 & 100.0 \\
$1.00 \mathrm{~mm}$ & 6.67 & 5.00 & 100.0 & 9.40 & 100.0 & 30.00 & 100.0 \\
\hline
\end{tabular}

attributed to two causes: attenuation of the incident X-ray beam before hitting the sample, and attenuation of the photoelectron signal along the path from the sample to the analyzer. Of course, in a general scenario, one would also have to consider the possibility of the formation of an adsorbent overlayer under high pressures which would mask some of the signal originating from the underlying sample, or indeed the possibility of chemical changes occurring at elevated pressures. However, for the study of a polycrystalline silver foil under a molecular nitrogen atmosphere at room temperature these effects can be safely ignored.

The effect of the attenuation of the incident X-ray beam in the gas phase is easily obtained by reference to photodiode measurements of the intensity of the X-ray spot at various pressures (Figure 8, adapted from the data published in reference [3]). In this paper, to eliminate the effect of the attenuation of the X-ray beam, all of the reported signal intensities have been normalized to the same actual incident X-ray intensity at the position of the sample. This procedure leaves attenuation of the photoelectron signal due to scattering of the photoelectrons in the gas phase as the only factor which causes the signal intensity at elevated pressures to be lower than that under high vacuum. As such, the intensity of any photoemission peak under high vacuum, $I_{S}$, can be treated as the "original" intensity, before any attenuation of the photoelectron beam has occurred, of the same peak recorded under an elevated pressure. Attention is now turned to the quantitative analysis of the attenuation.

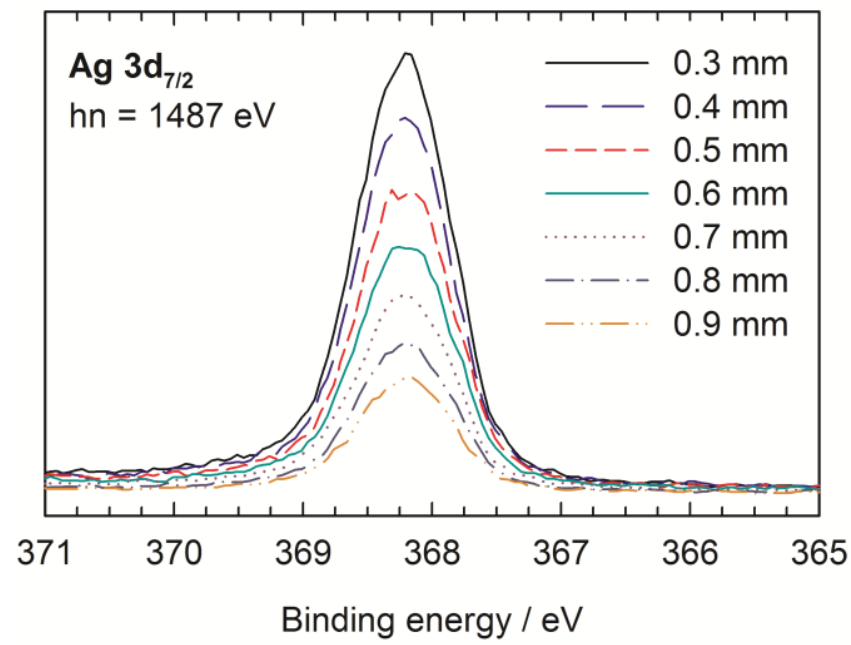

Figure 7: XPS spectra of a polycrystalline silver foil showing the $\mathrm{Ag} 3 \mathrm{~d}_{5 / 2}$ region, measured under a 5 mbar $\mathrm{N}_{2}$ atmosphere, as the separation between the sample and the first aperture of the differential pumping setup was varied. 


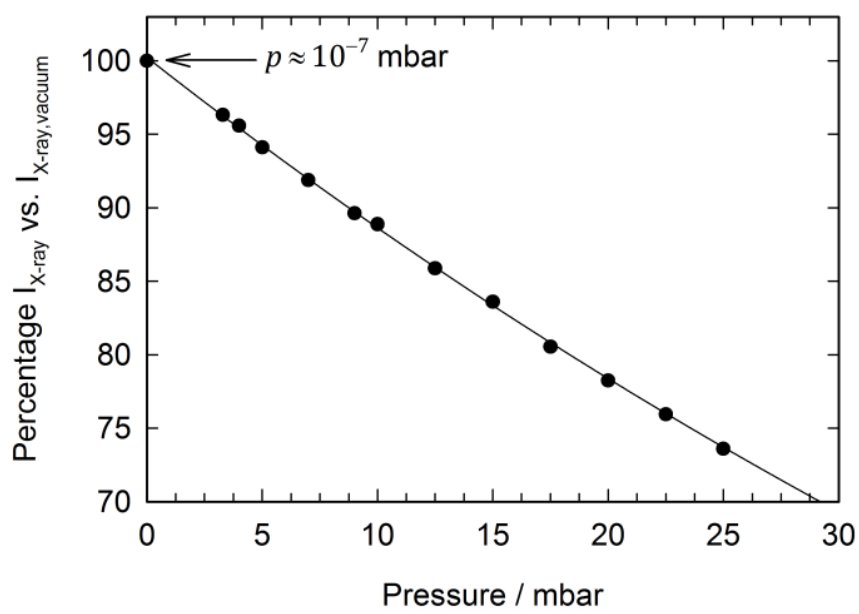

Figure 8: Intensity of the X-ray beam at the point of the sample, as a percentage of the intensity under conditions of high vacuum, plotted as a function of pressure in the analysis chamber.

For an infinitesimal path length, $d l$, the attenuation of the signal, $d I$, is given by the Beer-Lambert law in terms of the signal intensity, $I$, the concentration of the absorbing species, $C$, and the absorption coefficient of the absorbing species, $\alpha$ (Equation 9).

$$
d I=-\alpha c I d l
$$

To find an expression for the intensity of the signal that reaches the detector, as a function of the vacuum intensity, it is necessary to integrate Equation 9 across the entire path length of the photoelectrons, allowing for the fact that the exact pressure profile along this path is unknown. To simplify the problem, we consider this path to consist of two distinct regions, chosen to lie on either side position $l_{0}$, as shown in Figure 9 .

The position $l_{0}$ is chosen such that in region $1\left(l<l_{0}\right)$ the pressure remains effectively constant and equal to the pressure measured in the bulk of the analysis chamber, whereas in region $2\left(l>l_{0}\right)$ the pressure is in general a function of position. Furthermore, we treat all of the photoelectrons as though they travelled along the shortest straight line from the sample through the middle of the differential pumping apertures. This is justified by the relatively small angular acceptance of the instrument of $\pm 13^{\circ}$, limited by the size of the second aperture $\left(\cos \left(13^{\circ}\right) \approx 0.97\right)$.

Integrating Equation 9 across the path length of the photoelectrons from the sample $(l=s)$ to the detector $(l=f)$ gives:

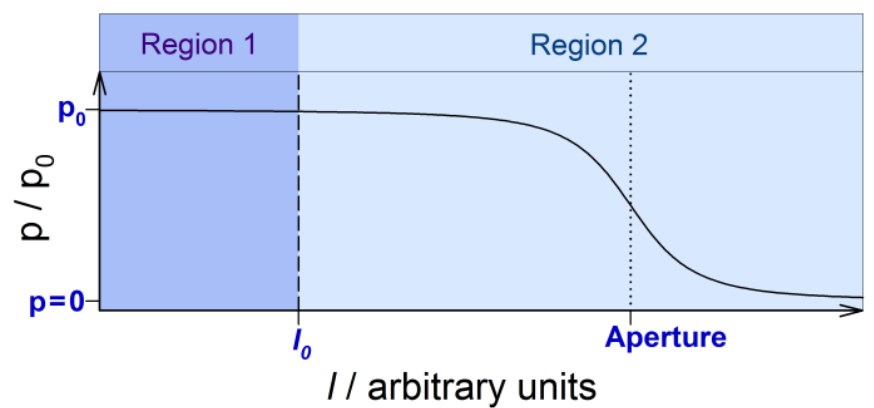

Figure 9: A schematic showing the integration regions used in Equation 10, overlaid on the theoretical pressure profile obtained from molecular flow simulations. The position $l_{0}$ is chosen such that in region 1 the pressure remains approximately equal to the pressure in the bulk of the analysis chamber. 


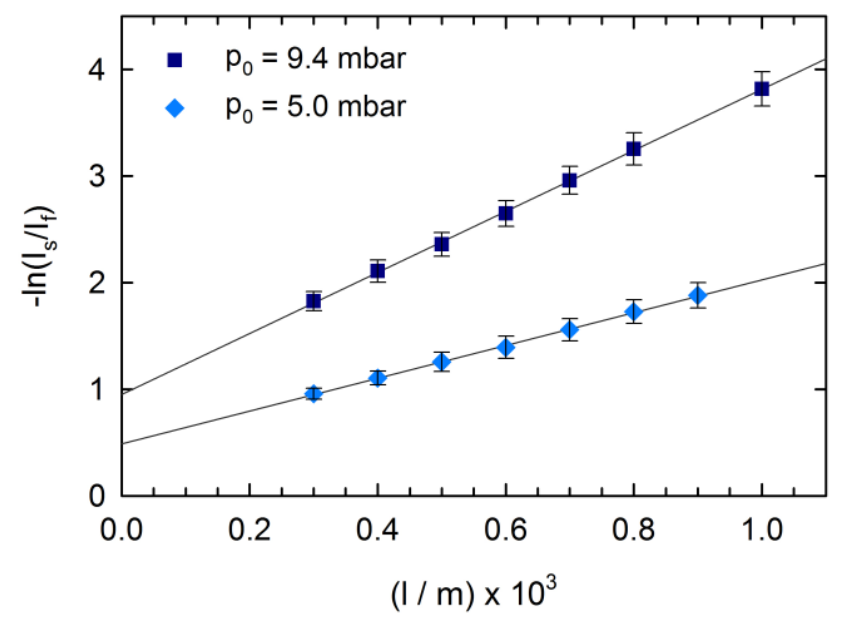

Figure 10: Negative logarithm of the intensity of the $\mathrm{Ag} 3 \mathrm{~d}_{5 / 2}$ photoemission peak, normalized to the intensity in high vacuum, plotted against distance between the sample and the first aperture of the differential pumping system. The data points for both 9.4 and 5 mbar lie on a straight line, indicating that in the region of the data points the pressure remains constant within the error of the measurements.

$$
\int_{s}^{f} d I=\int_{s}^{f}-\alpha p(l) I d l
$$

For convenience we use the pressure of the gas, $p$, instead of the concentration $c$, which is justified as long as the gas behaves approximately like an ideal gas. The proportionality constant for $p$ vs $c$ is absorbed into the attenuation coefficient $\alpha$. Provided the sample is located in region 1 , such that $s<l_{0}$, the right hand side can be rewritten as a sum of two integrals. By definition in region $1 p=p_{0}$.

$$
\int_{s}^{f} d \ln (I)=-\int_{s}^{l_{0}} \alpha p_{0} d l-\int_{l_{0}}^{f} \alpha p(l) d l
$$

If we first assume that the pressure profile at $l>l_{0}$ is independent of the position of the sample, the second term on the right hand side is independent of $s$ and can therefore be treated as a constant. Thus, defining $l_{0}-s=x$ as the coordinate of the sample along the principal axis:

$$
\ln \left(\frac{I_{f}}{I_{S}}\right)=-\alpha p_{0} x+C
$$

Accordingly, provided all of the sample positions are chosen in the region where the pressure is effectively constant, a plot of $-\ln \left(I_{f} / I_{S}\right)$ vs $x$ should give a straight line with a slope $\alpha p_{0}$. Such plots are shown for two different pressures in Figure 10, and it can indeed be seen, that the experimental data points fit well to a straight line in both cases (coefficient of determination > 0.999).

These results indicate that in the region more than one diameter of the aperture away from the first aperture the pressure does indeed remain constant within the error of the measurements. To estimate the magnitude of this error, alternative slopes were fitted between any two consecutive data points in the data series. Such an analysis yields a variance of up to $+-12 \%$, indicating that the present method would be insensitive to pressure fluctuations of a smaller magnitude. Nevertheless, these results provide supporting experimental evidence to the prediction that the pressure at distances greater than 1 diameter of the aperture from the aperture should be no less than ca $90 \%$ of the pressure at the back of the analysis chamber. 
Table 3 - A comparison of published scattering cross-sections for electrons in $\mathrm{N}_{2}$ gas

\begin{tabular}{cc}
\hline Source & Scattering cross-section \\
\hline This work & $1.26 \times 10^{-20} \mathrm{~m}^{2}\left(\mathrm{E}_{\mathrm{k}}=1116 \mathrm{eV}\right)$ \\
& $8.74 \times 10^{-21} \mathrm{~m}^{2}\left(\mathrm{E}_{\mathrm{k}}=1399 \mathrm{eV}\right)$ \\
Reference[16] & $1.87 \times 10^{-20} \mathrm{~m}^{2}\left(\mathrm{E}_{\mathrm{k}}=1200 \mathrm{eV}\right)$ \\
Reference[17] & $1.65 \times 10^{-20} \mathrm{~m}^{2}\left(\mathrm{E}_{\mathrm{k}}=1296 \mathrm{eV}\right)$ \\
& $1.49 \times 10^{-20} \mathrm{~m}^{2}\left(\mathrm{E}_{\mathrm{k}}=1444 \mathrm{eV}\right)$ \\
Reference[11] & $9.9 \times 10^{-21} \mathrm{~m}^{2}\left(\mathrm{E}_{\mathrm{k}}=886 \mathrm{eV}\right)$ \\
Reference[18] & $2.13 \times 10^{-20} \mathrm{~m}^{2}\left(\mathrm{E}_{\mathrm{k}}=1000 \mathrm{eV}\right)$ \\
\hline
\end{tabular}

According to Equation 12, the slopes of the lines in Figure 10 are equal to $\alpha p_{0}$. A plot of $\alpha p_{0}$ vs $p_{0}$ should also yield a straight line with a slope equal to the attenuation coefficient. In Figure 11, such plots are shown for two sets of data, one corresponding to the $\mathrm{Ag} 3 \mathrm{~d}$ core level photoelectrons emitted at a kinetic energy of $1116 \mathrm{eV}$, and the other corresponding to $\mathrm{Au} 4 \mathrm{f}$ core level photoelectrons emitted at a kinetic energy of 1399 $\mathrm{eV}$. Straight-line plots are found in each case, and as expected, the attenuation coefficient is found to decrease with increasing electron kinetic energy.

Alternatively, if it is assumed that attenuation of the photoelectron signal arises solely from gas phase ionization processes, Equation 9 can be rewritten in terms of the scattering cross-section, $\sigma$, and the number density of the gas atoms, $N_{g} / V$ (Equation 13). Following substitution of the ideal gas law and rearrangement (Equation 14) photoelectron scattering cross-sections in nitrogen are obtained for the two kinetic energies. These can be compared to literature values for the total (elastic + inelastic) scattering cross-sections for electrons of similar energy in nitrogen gas (Table 3). It should be noted that in the present case some elastically scattered electrons may still reach the detector, however, their contribution to the overall signal is expected to be minor due to the narrow acceptance angle of the analyzer.

$$
d I=-\sigma \frac{N_{g}}{V} I d l
$$

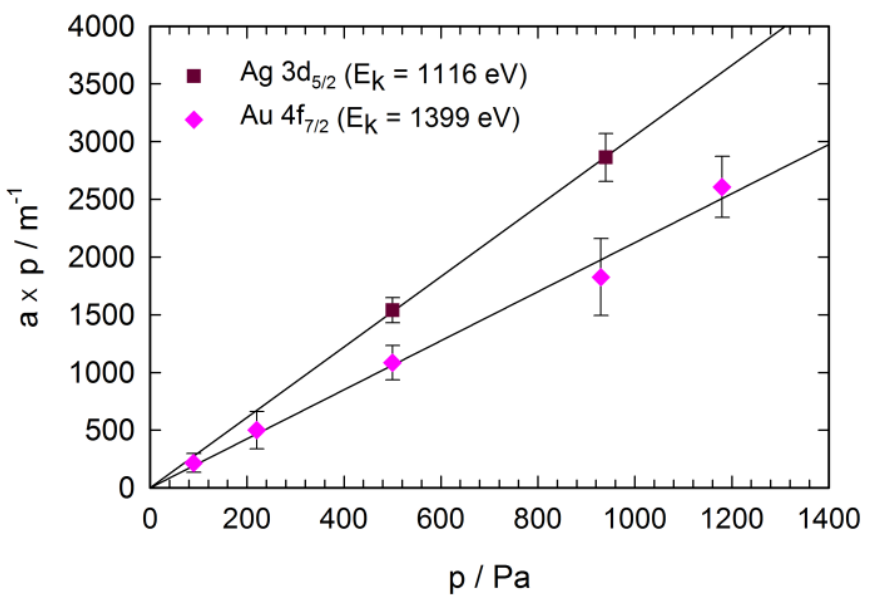

Figure 11: A plot of $\alpha p_{0}$ vs $p_{0}$ for measurements of both the $\mathrm{Ag} 3 \mathrm{~d}_{5 / 2}$ and the $\mathrm{Au} 4 \mathrm{~d}_{7 / 2}$ core levels corresponding to photoelectrons emitted with a kinetic energy of $1116 \mathrm{eV}$ and $1399 \mathrm{eV}$ respectively. As expected, the slope of the line, equal to the value of the attenuation coefficient, decreases with increasing electron kinetic energy. 


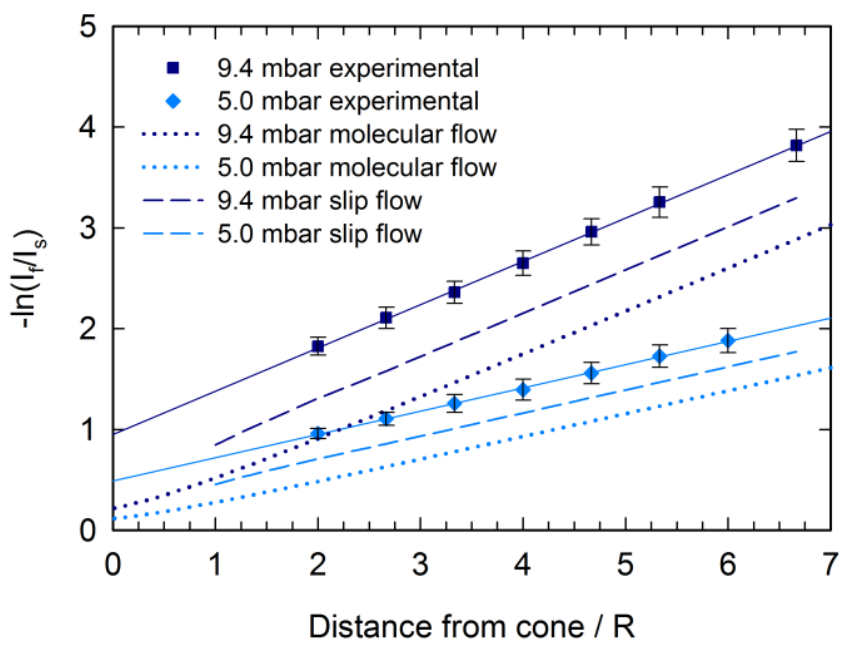

Figure 12: A comparison of the experimentally observed attenuation of the $\mathrm{Ag} 3 \mathrm{~d}_{5 / 2}$ photoemission signal at different pressures and sample positions with the theoretical curves for $-\ln \left(I_{f} / I_{s}\right)$ vs $d$ in units of $R$, obtained using the experimentally determined attenuation coefficient and either the pressure profiles from the slip-flow simulations or the molecular flow expression from reference [8].

$$
\sigma=\frac{\alpha R T}{N_{L}}
$$

Knowledge of the obtained attenuation coefficients allows us to obtain the "theoretical" curves for $I_{f} / I_{S}$ vs distance plotted in Figure 12 as dashed and dotted lines. For this, we have integrated Equation 9 over the path of the electron using either the molecular flow equation for the pressure profile, or the pressure profiles from the Star-CCM+ simulations, and the $\alpha$ value determined from Figure 10. For the molecular flow case, the integration can be performed analytically (Equations 15-16), whereas for the simulated pressure profiles it is done numerically, independently for every simulation corresponding to a different sample-to-cone distance. It should be emphasized here that this procedure is only meaningful as long as the value of $\alpha$ has been obtained without making any assumptions about the pressure profile in the region where $p<p_{0}$, as is the case in the present work. In Equations 15-16 the distance 1 is expressed in units of $\mathrm{R}$, where $\mathrm{R}$ is the radius of the $1^{\text {st }}$ aperture. For consistency the value of the attenuation coefficient $\alpha$ also needs to be expressed in units of $\mathrm{Pa}^{-1} \mathrm{R}^{-1}$.

$$
\begin{gathered}
\int_{s}^{f} d I=\int_{s}^{f}-\alpha p(l) I d l \approx \int_{s}^{\infty}-\alpha p(l) I d l \\
p(l)=\frac{1}{2} p_{0}\left(1-\frac{l}{\sqrt{1+l^{2}}}\right) \\
\int_{s}^{f} d \ln (I)=-\frac{1}{2} p_{0} \alpha \int_{s}^{\infty}\left(1-\frac{l}{\sqrt{1+l^{2}}}\right) d l \\
\ln \frac{I_{f}}{I_{s}}=-\frac{1}{2} p_{0} \alpha\left(\sqrt{s^{2}+1}-s\right)
\end{gathered}
$$


It is finally noted that the present analysis is reliant on the fact that the kinetic energy of the photoelectrons remains approximately constant in the region where there is a significant gaseous atmosphere, i.e. where a significant amount of signal attenuation occurs. This is true for the standard operation mode of the HiPP-2 analyzer, used for the measurements in this paper, as no electric fields are applied between the first and the second apertures. It is not true for the newly developed swift acceleration lens tables that have been optimized for maximum throughput [19].

From Figure 12 it is apparent that the molecular flow pressure profile vastly underestimates the amount of signal attenuation that occurs between the sample and the analyzer. The slip-flow simulations do considerably better, but the total amount of signal attenuation is still somewhat underestimated. There are three likely reasons for this. Firstly, in the simulation geometry perfect pumping is assumed for gas molecules that hit the boundaries beyond the first aperture. In practise, there is a likelihood that molecules may collide off the walls of the chamber or the second aperture cone, which is not accounted for in the simulations. The manometer in the first pumping stage in the experimental apparatus is mounted between the apertures and the turbo pump entrance so the pressure reading is likely an underestimate of the actual pressure at the second aperture. Secondly, it was noted previously that the procedure for experimentally determining the distance between the sample and the aperture always produces a slight underestimate of the actual distance, although the magnitude of the error should be much smaller than what would be required to account for the difference between the theoretical and experimental curves in Figure 12. Thirdly, it is assumed in the calculations that all electrons travel along the symmetry axis of the analyzer, whereas in practise the finite angular acceptance means that the paths of some electrons are longer resulting in greater signal attenuation.

\section{Conclusions}

We have studied the pressure variations around the sample position in a high-pressure photoelectron spectrometer using computational fluid dynamics modelling. Pressure profiles were simulated for the flow of nitrogen through an $0.3 \mathrm{~mm}$ aperture positioned at the end of a cone, at three different pressures between 5 and $30 \mathrm{mbar}$ and a range of different sample-to-cone distances. These simulations should provide a more reliable description of the pressure profiles than the equation derived under the assumption of molecular flow, since the assumption of molecular flow is invalid for pressures in the millibar range for flow through an $0.3 \mathrm{~mm}$ aperture. They are also indicative of the expected behaviour for other cone sizes but similar values of the Knudsen number. Additionally, XPS spectra were recorded at a series of distances under a 5 mbar and a 9.4 mbar $\mathrm{N}_{2}$ atmosphere and the attenuation of the signal was analyzed using the Beer-Lambert law.

To summarize the findings of the presented simulations, we note that at a working distance of 1 aperture diameter, the pressure at the sample surface was always no less than $\sim 95 \%$ of the pressure measured at the back of the chamber. A working distance of 2 diameters ensured that the pressure at the sample surface was at least $\sim 98.5 \%$ of the pressure at the back of the chamber, but typically more than $99 \%$. These values are broadly similar to the previous findings based on the molecular flow model. However, our calculations also indicate that there is a general tendency for the pressure drop at the aperture to become steeper, as the base 
pressure of the chamber is raised. For example, for a base pressure of $30 \mathrm{mbar}$ and a $0.3 \mathrm{~mm}$ cone, the calculated pressure at the sample surface is over $96 \%$ of the base pressure even if the sample-to-cone distance is just $0.15 \mathrm{~mm}$, whilst at $5 \mathrm{mbar}$, at the same working distance the pressure at the sample surface along the symmetry axis is just $\sim 84 \%$ of the base pressure, and there also exists a significant pressure gradient along the plane of the sample.

Finally, we hypothesize that under certain special circumstances it could actually be advantageous to deliberately measure at a working distance much shorter than the aperture diameter. A shorter sample-toaperture distance will in general mean a steeper pressure gradient along the symmetry axis, and the steeper the pressure gradient, the weaker the attenuation of the signal for a given actual pressure at the point of measurement. The use of an excitation source with a spot size that is much smaller than the aperture would also ensure that under the illuminated area, the pressure and temperature can be expected to be nearly constant, even if considerable variations exist across the whole sample. Further experimental and theoretical work is required, though, to verify whether this strategy would be practicable for extending the pressure range under which high-pressure photoelectron spectra can be recorded.

\section{Acknowledgements}

This work was supported by the Swedish Governmental Agency for Innovation Systems (VINNOVA). S.K.E, M.H, H.S, H.R. gratefully acknowledge StandUp for Energy, Swedish Energy Agency, the Swedish Research Council (Grant nos. 2012-4681, and 2012-4721) for financial support. D.J.P. acknowledges support from the Royal Society (UF100105). J.M.K. acknowledges support from the EPSRC for a Doctoral Prize Studentship. D.J.P and I.J.V.G acknowledge support from the EPSRC (EP/K004913/1 and EP/J021199/1).

\section{References}

1. Hüfner S. Photoelectron Spectroscopy: Principles and Applications, 3 edn. Springer: Berlin, 2010.

2. Starr DE, Liu Z, Havecker M, Knop-Gericke A, Bluhm H. Investigation of solid/vapor interfaces using ambient pressure X-ray photoelectron spectroscopy. Chem Soc Rev 42, 5833-5857 (2013).

3. Eriksson SK, Hahlin M, Kahk JM, Villar-Garcia IJ, Webb MJ, Grennberg H, et al. A versatile photoelectron spectrometer for pressures up to 30 mbar. Rev Sci Instrum 85, (2014).

4. Kaya S, Ogasawara H, Naslund LA, Forsell JO, Casalongue HS, Miller DJ, et al. Ambient-pressure photoelectron spectroscopy for heterogeneous catalysis and electrochemistry. Catal Today 205, 101105 (2013).

5. Siegbahn K. ESCA Applied to Free Molecules. North-Holland Publishing Company: Amsterdam, 1969.

6. Siegbahn H, Siegbahn K. ESCA applied to liquids. Journal of Electron Spectroscopy and Related Phenomena 2, 319-325 (1973).

7. Joyner RW, Roberts MW, Yates K. High-Pressure Electron Spectrometer for Surface Studies. Surf Sci 87, 501-509 (1979).

8. Ogletree DF, Bluhm H, Lebedev G, Fadley CS, Hussain Z, Salmeron M. A differentially pumped 
electrostatic lens system for photoemission studies in the millibar range. Rev Sci Instrum 73, 38723877 (2002).

9. Barber RW, Sun Y, Gu XJ, Emerson DR. Isothermal slip flow over curved surfaces. Vacuum 76, 73 81 (2004).

10. Atkins P, de Paula J. Physical Chemistry, 8 edn. Oxford University Press: Oxford, 2006.

11. Grunze M, Dwyer DJ, Nassir M, Tsai Y. Controlled-Atmosphere Photoelectron-Spectroscopy. Acs Sym Ser 482, 169-182 (1992).

12. Grass ME, Karlsson PG, Aksoy F, Lundqvist M, Wannberg B, Mun BS, et al. New ambient pressure photoemission endstation at Advanced Light Source beamline 9.3.2. Rev Sci Instrum 81, (2010).

13. Pantforder J, Pollmann S, Zhu JF, Borgmann D, Denecke R, Steinruck HP. New setup for in situ X-r ay photoelectron spectroscopy from ultrahigh vacuum to 1 mbar. Rev Sci Instrum 76, (2005).

14. Morris DL, Hannon L, Garcia AL. Slip Length in a Dilute Gas. Phys Rev A 46, 5279-5281 (1992).

15. Albertoni S, Cercignani C, Gotusso L. Numerical Evaluation of the Slip Coefficient. Physics of Fluids 6, 993 (1963).

16. Garcia G, Perez A, Campos J. Total Cross-Section for Electron-Scattering from N-2 in the EnergyRange 600-5000 Ev. Phys Rev A 38, 654-657 (1988).

17. Karwasz G, Brusa RS, Gasparoli A, Zecca A. Total Cross-Section Measurements for E(-)-Co Scattering - 80-4000 Ev. Chem Phys Lett 211, 529-533 (1993).

18. Itikawa Y. Cross sections for electron collisions with nitrogen molecules. J Phys Chem Ref Data 35, $31-53$ (2006).

19. Edwards MOM, Karlsson PG, Eriksson SK, Hahlin M, Siegbahn H, Rensmo H, et al. Increased photoelectron transmission in High-pressure photoelectron spectrometers using "swift acceleration". Nuclear Instruments and Methods in Physics Research Section A: Accelerators, Spectrometers, Detectors and Associated Equipment 785, 191-196 (2015). 